\title{
Spectral Analysis of Magnetometer Swing in High- Resolution UAV-borne Aeromagnetic Surveys
}

\author{
Callum Walter \\ Department of Geological Sciences and \\ Geological Engineering \\ Queen's University \\ Kingston, Canada \\ callum.walter@queensu
}

\author{
Alexander Braun \\ Department of Geological Sciences and \\ Geological Engineering \\ Queen's University \\ Kingston, Canada \\ braun@queensu.ca
}

\author{
Georgia Fotopoulos \\ Department of Geological Sciences and \\ Geological Engineering \\ Queen's University \\ Kingston, Canada \\ georgia.fotopoulos@queensu.ca
}

\begin{abstract}
Electromagnetic interference produced by multirotor UAVs has the potential to compromise the integrity of UAV-borne total magnetic field (TMI) measurements collected with high-resolution optically pumped magnetometers. One method to overcome this challenge involves suspending the magnetometer sensor below the zone of electromagnetic interference via a semi-rigid mount. The semi-rigid mount allows the magnetometer payload to freely move in the pitch and roll axes, while rigidly fixing the yaw of the magnetometer to that of the multi-rotor UAV. The swinging motions of the magnetometer suspended below the UAV have the potential to introduce periodic variations in the collected magnetic field data. Within this study, spectral analysis was applied to UAVborne TMI measurements to assess contributions to the signal from the swinging, semi-rigidly mounted magnetometer payload. Overall, it was concluded that when the magnetometer was placed outside the zone of electromagnetic interference created by the UAV, compensation and filtering was not required to achieve industry standard measurements. This result was due to the magnetometer swinging through the relatively low-amplitude geomagnetic field gradient. However, when the magnetometer was placed within the zone of UAVinduced electromagnetic interference, a periodic, highfrequency signal was apparent in the TMI measurements. This was determined to be caused by the swinging of the suspended magnetometer payload within the high-gradient electromagnetic field produced by the multi-rotor UAV. The periodic signal $(\sim 0.35 \mathrm{~Hz})$ was successfully identified and removed with a low-pass filter in the frequency domain, resulting in TMI measurements of industry standard quality. Filtering is a necessary step to avoid contaminating the magnetic field signals originating from sub-surface targets with unwanted signals related to the swinging of the magnetometer. Filtering can be applied when the targeted signal frequencies and the swinging signal frequencies do not spectrally overlap. This relationship must be considered in order to avoid removing important target signals during the filtering process.
\end{abstract}

Keywords- Unmanned Aerial Vehicles, UAV Magnetic Surveys, Multi-rotor UAV, Magnetometry, Magnetic Interference, Noise Filtering, Spectral Analysis

\section{INTRODUCTION}

The development of commercially available multi-rotor unmanned aerial vehicles (UAVs) over the past decade has led to numerous sensors and payload systems being integrated onboard these platforms. One of these innovative sensors includes high-resolution, optically pumped magnetometers. These magnetometer payloads are dominantly used in geophysical surveys for mineral exploration and infrastructure detection applications [1]. Three main payload integration methods have been developed that allow for the collection of high-resolution UAV-borne magnetic data. These methods are: (i) suspending the magnetometer via a semi-rigid boom below the UAV [2 \& 3], (ii) suspending the magnetometer via a cable and an aerodynamic casing below the UAV [4], and (iii) fixing the magnetometer to a rigid boom in front, behind, or directly below the UAV [5 \& 6]. Each method attempts to balance three critical design considerations. These include: (i) reducing electromagnetic interference emanating from the UAV, (ii) keeping the attitude of the optically pumped magnetometer stable during flight, and (iii) balancing the weight of the payload system on the UAV [2].

Presently, one of the most widely used integration methods for conducting UAV-borne magnetometry surveys in Canada is method (i), semi-rigidly suspending the magnetometer below the UAV. This method will henceforth be the focus of this investigation. The semi-rigid mount is effective due to its relatively simple design and few physical interactions required to functionally balance the three design considerations [ $2 \& 7]$. To maintain industry quality TMI measurements, the semi-rigid mount must extend the magnetometer outside the region of electromagnetic interference that is generated by the multi-rotor UAV in flight. For a DJI S900, the distance below the UAV where the electromagnetic interference has attenuated below the sensitivity threshold $(0.1 \mathrm{pT} / \mathrm{Hz})$ of an optically pumped magnetometer is $\sim 3 \mathrm{~m}$ [3 \& 8]. However, this multi-rotor UAV has a relatively low flight endurance of approximately 15 minutes when carrying the $2.2 \mathrm{~kg}$ suspended magnetometer payload [7]. As UAV platforms with larger payload capacities and flight endurances enter the market, there will be a transition to exploit their increased surveying efficiency. The challenge this poses for UAV-borne magnetometry is that the larger and heavier multi-rotor UAV platforms will produce larger regions of electromagnetic interference. These regions have the potential to be sensed by the magnetometer at greater distances below the UAV. This advancement in platform endurance has the potential to compromise the integrity of the collected TMI data via a semi-rigid mount for two reasons: (i) the magnetometer may be positioned within the zone of electromagnetic interference from the larger UAV, or (ii) placing the magnetometer outside the larger zone of electromagnetic interference may cause excessive swinging in the magnetometer. This has the potential to either degrade flight efficiency of the system or cause the magnetometer to become unlocked with the magnetic field, voiding measurements. To date, little research has been conducted to quantify the signal produced by a swinging, semi-rigidly suspended magnetometer in flight below a multi-rotor UAV. Herein, the time and frequency domain of UAV-borne TMI data are investigated using spectral analysis. TMI data were collected both within and outside the zone of electromagnetic interference produced by the multi-rotor UAVs.

\section{MATERIALS \& METHOD}

Two heavy-lift multi-rotor UAV platforms (a DJI S900 and a DJI Wind 4) were used to collect the UAV-borne TMI 
data. Both were equipped with autonomous flight controllers (A2 \& A3, respectively) and were compatible with the DJI Ground Station software. This allowed for autonomous flights to be conducted at a speed of $\sim 10 \mathrm{~m} / \mathrm{s}$, along $500-$ $1500 \mathrm{~m}$ long lines. The flight elevation used for each survey was approximately $35 \mathrm{~m}$ above the ground. The magnetic sensor used throughout all tests was a GEM Systems Inc. GSMP-35U potassium vapor magnetometer. It is composed of an electrical console and a magnetometer sensor, weighing $2.2 \mathrm{~kg}$ altogether. The magnetometer electrical console was secured directly to the frame of the multi-rotor UAV. However, the magnetometer sensor, used to passively measure the TMI, was semi-rigidly mounted to the UAV and suspended $\sim 3-5$ meters below each UAV platform, as demonstrated in Figure 1. All on-board sensors collected data at or above $10 \mathrm{~Hz}$ resulting in an observation density of approximately one point per meter along the flight lines.

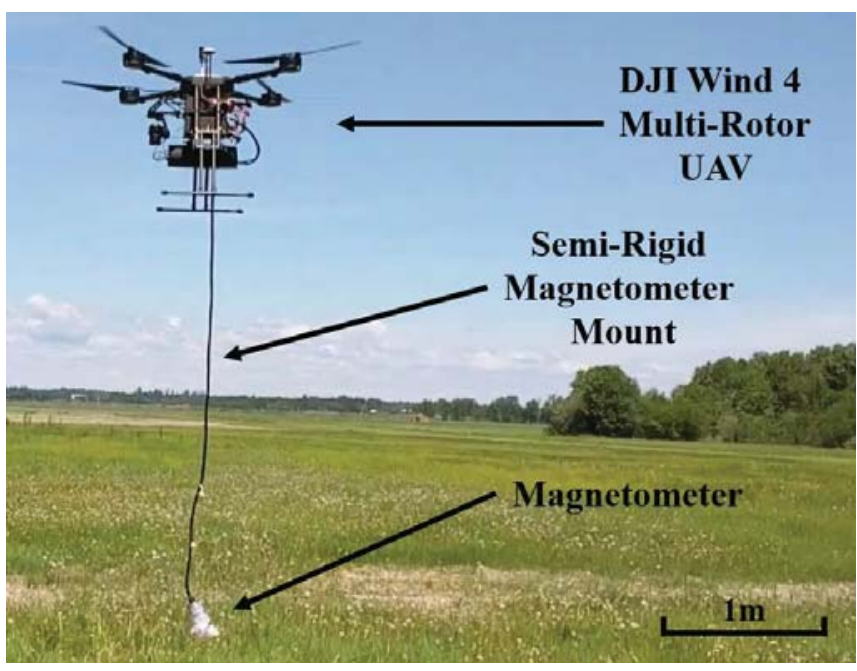

Figure 1: DJI Wind 4 data acquisition system and semirigidly mounted potassium vapor magnetometer during a test flight of the system on June 21, 2018.

The periodic swinging of the magnetometer below the UAV in $3 \mathrm{D}$ space was approximated to be an imperfect pendulum. The estimated frequency of this procession was calculated to be between $0.4-0.2 \mathrm{~Hz}$, using cable lengths ranging from $3-5$ meters. Equation 1 estimates the swinging frequency $f$, where $L$ is the length of the pendulum cable and $g$ is the force of gravity.

$$
f=1 /(2 \pi \sqrt{ }(L / g))
$$

Following data acquisition, the TMI measurements were processed to calculate the residual magnetic field intensity (RMI) in accordance to industry standards and were visualized in time domain, frequency domain, and spectrogram plots. The RMI data were analyzed to determine if signal variations within the expected bandwidth of $0.4-0.2 \mathrm{~Hz}$ occurred. An RMI dataset that had a measurable signal within this bandwidth was crosscorrelated with data collected from an inertial measurement unit (IMU) that was fixed to the semi-rigid mount. If the measured frequency of the swinging semi-rigid mount matched the frequency observed in the RMI data, the RMI data were low-passed within the frequency domain to remove this component of the signal.

\section{RESULTS}

\section{A. Case 1 - DJI S900 Multi-rotor UAV, 3 m Suspension}

The UAV-borne aeromagnetic survey conducted within Case 1 used a DJI S900 multi-rotor UAV and a magnetometer offset distance of $\sim 3 \mathrm{~m}$ below the airframe. This offset distance was chosen based on the work of Parvar (2016), where the magnetic field produced by a DJI S900 multi-rotor UAV was shown to attenuate sufficiently at a $3 \mathrm{~m}$ distance. Analysing the collected RMI data in the time domain revealed that no measurable UAV-induced electromagnetic interference was sensed by the magnetometer within the expected swinging bandwidth or at higher-frequencies above $0.4 \mathrm{~Hz}$, as shown in Figure 2.

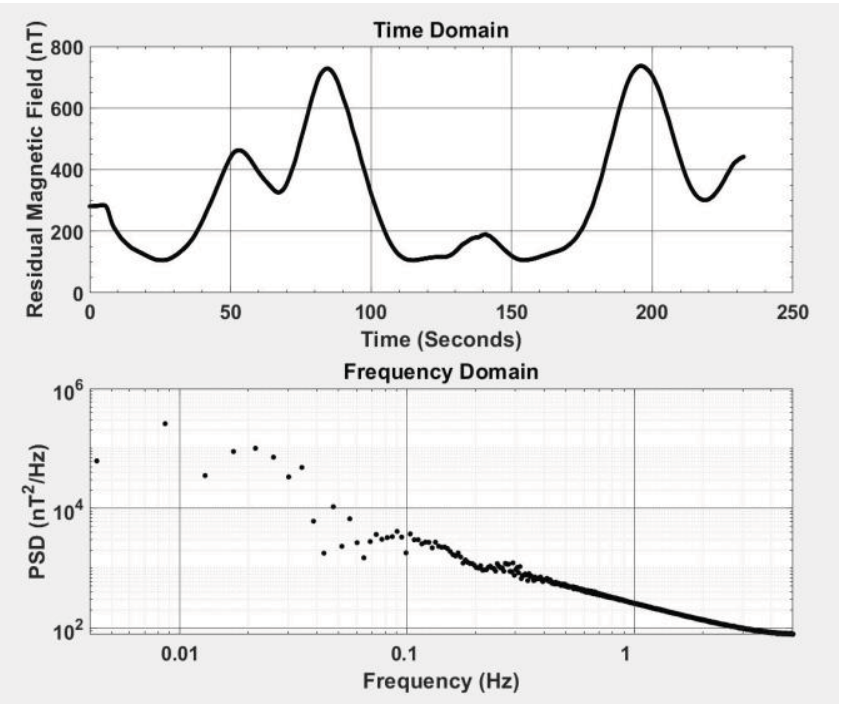

Figure 2: Case 1: TMI data collected $3 \mathrm{~m}$ below the DJI S900 Multi-rotor UAV, where the magnetometer is outside the electromagnetic influence of the platform.

However, upon close inspection of the data in the frequency domain, a small increase in the power spectral density (PSD) is present in the $0.4-0.2 \mathrm{~Hz}$ bandwidth. This was further analysed by identifying when the signals in the $0.4-0.2 \mathrm{~Hz}$ bandwidth (frequency domain) occurred throughout the survey (time domain) using a spectrogram plot. It was determined that the $0.4-0.2 \mathrm{~Hz}$ signals were the result of the magnetometer swinging at the end of the survey lines, as the UAV rounded each $180^{\circ}$ corner. This flight manoeuvre caused the most agitation in the suspended magnetometer due to the rapid changes in acceleration and attitude. This swinging-related RMI signal was recorded at less than $1 \mathrm{nT}$ peak-to-peak and was not significant in amplitude compared to the dominant geological signals of $700 \mathrm{nT}$ within the survey area. A second occurrence of $0.4-$ $0.2 \mathrm{~Hz}$ signals was also observed in the dataset. These occurred as the UAV traversed regions with a relatively high horizontal geomagnetic field gradient at the edges of the geologic targets at the site. At these locations, the swinging of the magnetometer caused an amplitude change of $0.5 \mathrm{nT}$, peak-to-peak. These observations confirm that suspending an optically pumped vapour magnetometer $\sim 3 \mathrm{~m}$ below a DJI S900, with a $2.2 \mathrm{~kg}$ payload, places it outside the zone of UAV-induced electromagnetic interference. This resulted in no significant signal contributions within the $0.4-0.2 \mathrm{~Hz}$ bandwidth (expected swinging frequencies) within the recorded TMI data. 


\section{B. Case 2-DJI Wind 4 Multi-rotor UAV, 3m Suspension}

The UAV-borne aeromagnetic survey conducted within Case 2 used a DJI Wind 4 multi-rotor UAV and a magnetometer offset distance of $\sim 3 \mathrm{~m}$ below the airframe. At this distance, it was assumed that the electromagnetic field produced by a DJI Wind 4 would be sensed by the magnetometer. The reason for this is two-fold: (i) the Wind 4's brushless DC electromagnetic motors are significantly larger and are therefore able to produce more powerful alternating electromagnetic fields, and (ii) the total platform weight increased by $5 \mathrm{~kg}$ compared to Case 1 , causing the UAV to produce an increased amount of power to maintain flight. These two factors both cause the DJI Wind 4 UAV to produce a larger $3 \mathrm{D}$ region of electromagnetic interference.

Analyses of the collected RMI data revealed that there was significant platform-induced electromagnetic interference sensed by the magnetometer. The time and frequency domain plots in Figure 3 demonstrate this periodic signal which is centred around $\sim 0.35 \mathrm{~Hz}$. The observed swinging frequency of $\sim 0.35 \mathrm{~Hz}$ is slightly higher than the expected swinging frequency derived from Equation (1) for a $3 \mathrm{~m}$ long pendulum $(\sim 0.29 \mathrm{~Hz})$. This is likely caused by three factors: (i) the magnetometer is not a perfect pendulum, (ii) the swinging of the magnetometer is not confined to a $2 \mathrm{D}$ plane and (ii) there is an additional energy source besides gravity in the system, which is the forward motion of the magnetometer through the air. The measured swinging frequency was also correlated with the IMU that was placed on the semi-rigid mount $1 \mathrm{~m}$ above the magnetometer. From these results, Equation (1) gave a reasonable estimate of the measured swinging frequency of the magnetometer.

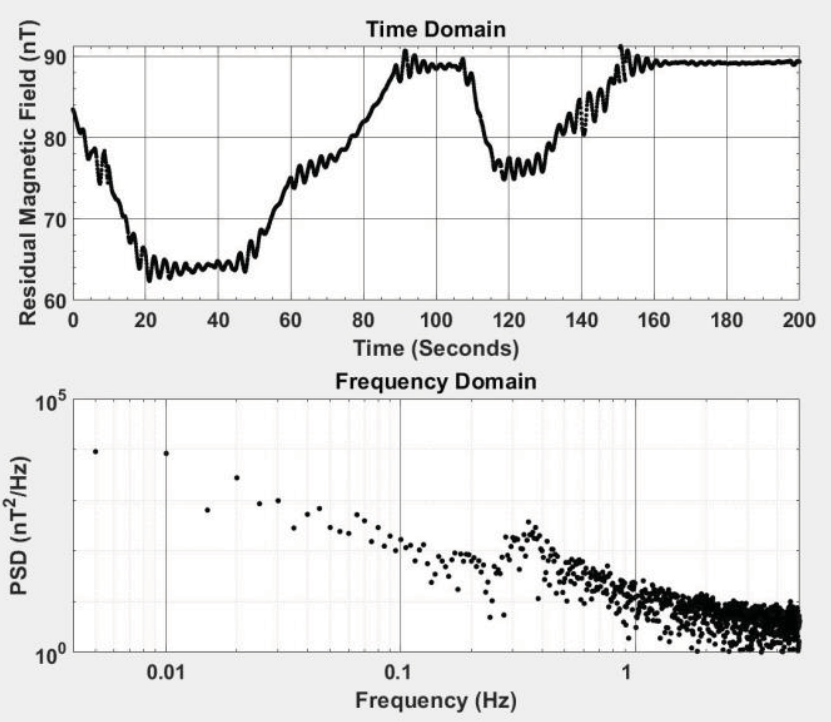

Figure 3: Case 2: TMI data collected $3 \mathrm{~m}$ below the DJI Wind 4 Multi-rotor UAV where the magnetometer is within the electromagnetic influence of the platform.

A low-pass Butterworth filter, with a cut-off frequency of $0.2 \mathrm{~Hz}$, was applied within the frequency domain to preserve the lower frequencies likely related to geological targets. The results of applying this filter are presented in Figure 4, where the periodic swinging related signal has been removed.

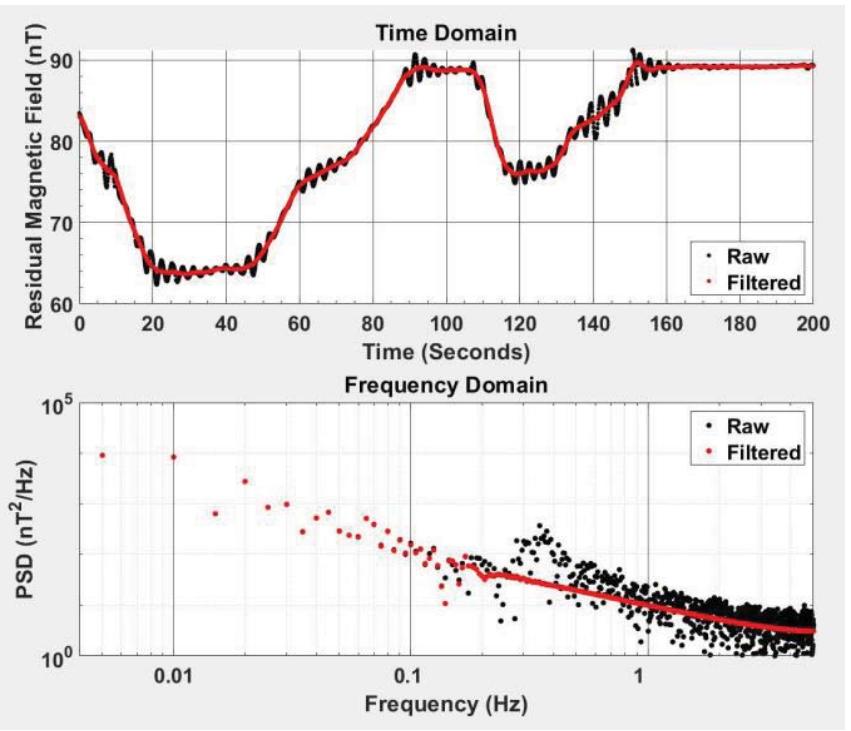

Figure 4: Low-pass filtered TMI data preserving the frequencies below $0.15 \mathrm{~Hz}$, collected from Case 2 .

\section{Case 3-DJI Wind 4 Multi-rotor UAV, $5 m$ Suspension}

The UAV-borne aeromagnetic survey conducted within Case 3 used a DJI Wind 4 multi-rotor UAV and a magnetometer offset distance of $\sim 5 \mathrm{~m}$ below the airframe. This offset distance was determined based on a series of platform-related tests conducted within this study. These tests demonstrated that the electromagnetic field produced by a DJI Wind 4, under load, was not sensed by the GSMP-35U magnetometer at a $5 \mathrm{~m}$ distance away from the motors. The time and frequency domain plots in Figure 5 confirm this through flight, revealing little swinging related signal amplitude in the estimated $0.4-0.2 \mathrm{~Hz}$ bandwidth.

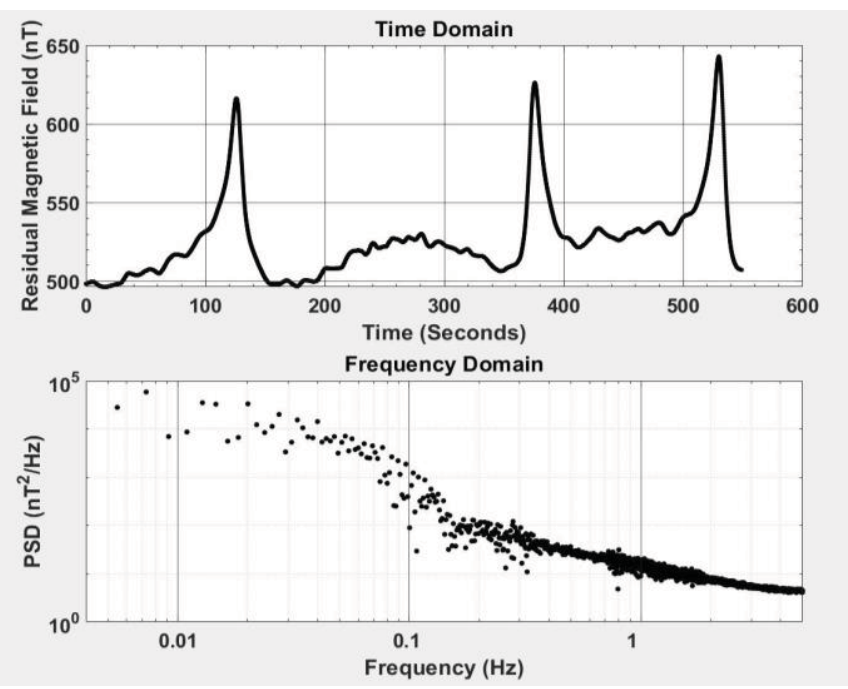

Figure 5: TMI data collected $5 \mathrm{~m}$ below the DJI Wind 4 Multi-rotor UAV where the magnetometer is outside the magnetic influence of the platform.

After correlating the time domain and frequency domain signals using a spectrogram, it was confirmed that there was a small amount of signal present within the $0.4-0.2 \mathrm{~Hz}$ bandwidth, with an amplitude between 0.5 - 1 nT peak-topeak. This signal is centered around a slightly lower frequency of $0.3 \mathrm{~Hz}$, compared to Case $1 \& 2$, due to the longer cable length of $5 \mathrm{~m}$. Again, the observed swinging in the signal was primarily the result of the same two 
occurrences concluded in Case 1: (i) the magnetometer was swinging with a large relative amplitude at the end of the survey lines, as the UAV rounded each $180^{\circ}$ corner, and (ii) the magnetometer was swinging a standard amount through areas where the horizontal geomagnetic field gradient was relatively high at the edges of anomalies. This confirms that the technique of suspending an optically pumped vapour magnetometer $5 \mathrm{~m}$ below a standard DJI Wind 4, with a 2.2 $\mathrm{kg}$ payload, is a viable solution to mitigate electromagnetic interference generated by the UAV. The results of Case 3 compliment the results of Case 1, even though Case 3 was flown with a different platform, cable length, and at a different location. This demonstrates the versatility and robustness of suspending the magnetometer outside the zone of electromagnetic interference generated by the UAV.

\section{DISCUSSION}

Within this study, the RMI signals produced by a swinging, semi-rigidly suspended magnetometer where quantified, given a range of magnetometer offset distances below a multi-rotor UAV. To achieve industry standard RMI measurements while surveying, one of two conditions needs to be met: (i) the magnetometer needs to be suspended outside the zone of electromagnetic interference produced by the UAV platform (Cases 1 \& 3), or (ii) when the magnetometer is suspended within the zone of electromagnetic interference, the RMI measurements need to be low-pass filtered to remove the swinging component of the signal (Case 2). For the second condition, the frequencies of the targeted geologic signals must also not spectrally overlap with the frequencies of the swinging signals being removed by the low-pass filter.

Cases 1 and 3 demonstrated that suspending the magnetometer outside the region of electromagnetic interference produced by a multi-rotor UAV can result in industry standard RMI measurements. This was confirmed for two different UAV models at two unique offset distances throughout the study. For Case 1, the offset distance between the optically pumped magnetometer and the DJI S900 multirotor UAV was confirmed to be $\sim 3 \mathrm{~m}$. For Case 3 , the offset distance below the DJI Wind 4 multi-rotor UAV was confirmed to be $\sim 5 \mathrm{~m}$. Overall, this demonstrates that using a semi-rigid mount to suspend the magnetometer below the UAV is a viable solution if the correct offset distance is determined and applied for each unique multi-rotor UAV.

Case 2 demonstrated that low-pass filtering the RMI data can remove signals related to the swinging of the magnetometer when it is positioned within the zone of electromagnetic interference produced by the UAV. This low-pass filtering method could be applicable for larger multi-rotor UAVs, where suspending the magnetometer at distances greater than $5 \mathrm{~m}$ may become impractical. Yet, within Case 2, the filtered RMI data were not directly validated with independent measurements gathered from outside the UAV's zone of electromagnetic interference. A more robust and complete conclusion would be to directly compare the low-pass filtered RMI data with independently observed RMI observations that do not include the UAV's electromagnetic interference. This full comparison would determine if low-pass filtering the UAV-induced electromagnetic interference is a truly viable solution to compensate the UAV-borne RMI measurements when the magnetometer is placed within the UAV's magnetic field.

\section{CONCLUSIONS}

In this study, spectral analysis was applied to RMI measurements collected via multi-rotor UAVs to assess the noise contribution of a swinging magnetometer payload. When the magnetometer was suspended outside the zone of electromagnetic field interference produced by the UAVs, there were negligible signals within the expected swinging frequency bandwidth $(0.4-0.2 \mathrm{~Hz})$. Therefore, suspending the magnetometer outside of each platform's unique zone of electromagnetic interference was proven to be a viable integration method. A low-pass filter was able to effectively remove signals within the expected swinging frequency bandwidth when the magnetometer remained within the zone of electromagnetic interference. Filtering could be a viable option for larger UAVs where the required suspension length cannot be achieved. In order to ensure that the filtering method does not remove targeted geological signals, the surveying speed of the UAV needs to be known. This allows both the wavelength and frequency of the geological targets and the swinging effect of the magnetometer to be analysed and separated. Pre-emptively changing the surveying speed of the UAV can help ensure that the recorded geological signals do not spectrally overlap with the swinging signals of the magnetometer and are therefore not removed by the lowpass filter.

\section{ACKNOWLEDGMENTS}

The authors thank Sumac Geomatics Inc. for providing funding through a MITACS Accelerate grant, piloting expertise and access to their facilities. NSERC and OGS are acknowledged for providing scholarships to CW. GEM Systems Inc. is greatly acknowledged for providing a GSMP-35U magnetometer.

\section{REFERENCES}

[1] A. Malehmir, L. Dynesius, K. Paulusson, A. Paulusson, H. Johansson, M. Bastani, M. Wedmark \& P. Marsden, "The potential of rotarywing UAV-based magnetic surveys for mineral exploration: A case study from central Sweden." The Leading Edge, 36, 7, 552-557, 2017.

[2] C. Walter, A. Braun \& G. Fotopoulos, "Impact of 3-D attitude variations of a UAV magnetometry system on magnetic data quality," Geophysical Prospecting, 1-15, 2018.

[3] K. Parvar, "Development and Evaluation of Unmanned Aerial Vehicle Magnetometry Systems," M. A. Sc. Thesis, Queen's University, Kingston, 1-141, 2016.

[4] GEM Systems Inc. Technical Staff, “AirBIRD UAV Magnetometer System," GEM Systems Inc., 2016.

[5] M. Cunningham, C. Samson, A. Wood \& I. Cook, "Aeromagnetic Surveying with a Rotary-Wing Unmanned Aircraft System: A Case Study from a Zinc Deposit in Nash Creek, New Brunswick, Canada," Pure And Applied Geophysics, 175, 9, 3145-3158, 2018.

[6] L. Tuck, C. Samson, J. Laliberté, M. Wells \& F. Bélanger, "Magnetic interference testing method for an electric fixed-wing unmanned aircraft system," Unmanned Vehicle Systems, 6, 3, 177-194, 2018.

[7] C. Walter, A. Braun \& G. Fotopoulos, "Integrating a Potassium Vapour UAV Magnetometer with a Multi -Rotor UAV Towards Industry Standard Airborne 3-D Magnetic Gradiometry." In Proc. Canadian Exploration Geophysical Society Symposium, Toronto, 2017.

[8] GEM Systems Inc. Technical Staff, "GSMP-35U User Manual V8.0," GEM Systems Inc., 2016.

[9] M. Cunningham, "Aeromagnetic surveying with unmanned aircraft systems.” M. Sc. Thesis, Carleton University, Ottawa, 1-156, 2016. 